\title{
Vehicular optical ranging and communication system
}

\author{
Zening Li, Linchao Liao, Albert Wang and Gang Chen*
}

\begin{abstract}
In this paper, we developed a ranging and communication system for vehicular safety applications. The light-emitting diode (LED) array and photodetector (PD) array serve as the transceiver. The imaging technique is applied to the receiver, which can detect the angle of incident light and imaged different lights to corresponding PDs. Benefiting from this structure, this system is able to detect distance as well as location, and work between multiple users simultaneously. While keeping a low cost and compact size, it can still provide high lateral resolution, high frame rate, and interference rejection performance.
\end{abstract}

Keywords: Intelligent transportation systems; Optical; Ranging; Communication

\section{Introduction}

As wireless technologies evolve at a rapid rate, opportunities are provided by these technologies to support more advanced vehicle safety applications. With the help of these technologies, vehicle-to-vehicle and vehicle-toinfrastructure communications become feasible. And this also offers the potential to support vehicle safety applications, such as traffic signal violation warning, curving speed warning, emergency electronic brake light, pre-crashing sensing for cooperative collision mitigation, cooperative forward collision warning system, left turn assistance, lane change warning, and stop sign movement assistance [1].

Most of these applications are based on radio frequency (RF) technologies, in particular the dedicated short-range communications (DSRC) at $5.9 \mathrm{GHz}$ [2], due to their significant advantages like very low latency communication and broadcasting ability [1]. However, RF has limited available bandwidth and may cause significant broadcast problem since the RF signal is usually transmitted in all directions. Furthermore, the RF ranging method cannot provide high lateral resolution because of its wide beamwidth, which can result in location ambiguity.

Nowadays, there has been a trend that more and more automobile manufacturers are using light-emitting diodes

${ }^{*}$ Correspondence: gachen@ee.ucr.edu

Department of Electrical Engineering, University of California, Riverside, 900 University Ave, Riverside, CA 92521, USA
(LEDs) as the light source due to their high efficiency, low cost, and fancy styling. This provides potential to emit ranging and communication signal with these LEDs. A lot of work has been done separately in LED optical ranging or communication for vehicular applications. In [3], the distance was calculated from the geometrical transformation of the position of LED markers mounted on the vehicle with an imaging-based technique. A low-cost LED ranging sensor for collision avoidance was designed in [4], which measures the time of flight of an optical pulse to determine the distance. Also, another time-offlight optical ranging system was proposed in [5], using a two-dimension imaging sensor to offer better lateral resolution. For vehicular optical communication, Cui et al. proposed an outdoor line-of-sight (LOS) visible light communication (VLC) path loss model and validated it by measurements [6]. In [7], the VLC channel models for intelligent transportation system application reflecting practical conditions were simulated. The effects of sunlight and other active VLC transmitters were also examined in [8].

For efficiency consideration, there is no need of two separated systems. And the integrated system of optical ranging and communication features the following advantages: (1) cooperative safety application becomes possible, and (2) the physical position of each node can be used to optimize networking. Nevertheless, a limited number of publications considered to combine the optical ranging and communication together. Mizui et al. proposed a

\section{Springer}

(c) $2015 \mathrm{Li}$ et al. This is an Open Access article distributed under the terms of the Creative Commons Attribution License (http:// creativecommons.org/licenses/by/4.0), which permits unrestricted use, distribution, and reproduction in any medium, provided the original work is properly credited. 
vehicle-to-vehicle communication and ranging system by multiplying the bit information to the spread spectrum (SS) code and measuring the phase shift of the SS code [9]. However, this system can only measure distance instead of acquiring the location information. In addition, it can operate with one target only. In order to get both the distance and location information, at least two receivers are needed [10]. In [11], a multi-user ranging and communication scheme was proposed, which makes this system be operated between multiple vehicles simultaneously. Techniques like frequency hopping and code hopping are also developed to reduce the SS code collision probability $[12,13]$.

In this paper, we modified the system proposed in [9], exploiting the LED and photodetector (PD) array as the transmitter and receiver, instead of a laser and single PD. This improved system is capable of working between multiple vehicles simultaneously acquiring both distance and location. Because of the good lateral resolution provided by the imaging-based receiver, it can greatly reduce the user interference and noise with a wide field of view (FOV). A scheme is proposed to mitigate the SS code collision without changing the hardware of the system.

The rest of this paper is organized as follows: The system design consideration is given in Section 2. Section 3 introduces the principle of the proposed combined system and the vehicular network. The experiment results for the basic ideas are presented in Section 4. At last, Section 5 concludes the work of this paper.

\section{System design consideration}

\subsection{Using LED as the transmitter}

In most conventional ranging systems, lasers or laser diodes (LDs) are usually used as the transmitter. Lasers and LDs can output a higher energy beam, compared to LEDs, which means they can be operated at a longer range. However, lasers and LDs are costly to acquire and maintain. While LEDs can offer an acceptable performance with a small fraction of cost.

Compact size is another advantage of LEDs. And lasers and LDs require more drive current to produce higher peak power, which makes the peripheral circuits for modulation usually complicated.

However, the wide beam angle is the main drawback of LEDs. In contrast to the beam angle of lasers or LDs, which is usually several milliradians, that of LEDs with a narrow beam is about 0.1 radians. This will bring in two problems: (1) the power density (power/area) decreases quickly as the measuring distance increases, and (2) lateral resolution is very low. We can see from the propagation function (Eq. 1) that the power intensity diminishes quickly over distance [14].

$$
I_{\text {target }}=\frac{4 \tau_{a} P_{t}}{\pi \theta_{t} R^{2}}
$$

where $I_{\text {target }}$ is the beam intensity on the target, $\tau_{a}$ is the atmospheric transmission loss, $P_{t}$ is the LED transmitted power, $\theta_{t}$ is the LED beam angle, and $R$ is the target distance. Collimating the LED beam is one possible way to mitigate this problem. But the collimation procedure is very difficult because of the LED core sharp. It requires a large diameter lens, which is not feasible for the LED array.

Another way is to address this problem at the receiver end. We will give details in the next section.

\subsection{Using the PD array as the receiver}

By employing a narrow beam laser or LD as the light source, the conventional ranging systems feature good lateral resolution. In order to produce a full view of the scene ranging data, a precise scanning mechanism is essential. On the other hand, for communication purposes, a tracking algorithm should be proposed to track the laser head and maintain the link. This is usually difficult in a moving situation.

Instead of constraining the beam width, limiting the receiver FOV is another approach to increase the ranging lateral resolution. The imaging lens makes it possible by providing different narrow FOVs to the elements of the PD array as the receiver sensor [15]. It can capture the entire scene while making the receiving process highly directional. The incoming light from different directions will be imaged to corresponding PDs as long as they can be separated wide enough in the real space. This will also help reduce the ambient noise since we are only interested in several certain PDs sensing high signal power. The imaging-based receiver allows distance measurement without scanning, while offering high lateral resolution and frame rate.

For the communication application, these PDs not only give our system great potential to increase the data rate but also enable our system to distinguish one user from another since they will be imaged to different PDs. This also provides a very effective media access control (MAC) for the future vehicular optical network since different users can transmit simultaneously without disturbing each other.

Increasing the number of PD will absolutely increase the resolution, but it will also increase the requirement of the data bus since it needs higher readout speed. Because only a few PDs are receiving optical signal, we need a tracking algorithm to find these PDs [16]. More PDs mean that a more complicated algorithm is needed. This is a tradeoff when designing the system, so we need to choose the number based on the application requirement.

\subsection{Spread spectrum waveform}

Even though the imaging-based method can greatly reduce the interference between users in the LOS 
communication link, there still exists the possibility that the backscattered signal could interrupt another user. Also, the signal could be corrupted by the noise. The spread spectrum (SS) code is robust against multiuser and noise. Employing the pulsed SS waveform as the ranging signal can reach a high peak power, which will bring a higher signal-to-noise ratio (SNR) and provide a longer operation distance, while maintaining a relative low average power. Low average power is crucial to eye safety which is usually the limit of the optical ranging system. And the optical communication system will also benefit from the SS code on rejecting the multiuser interference. There are several SS codes we could use in our system, and the performance may vary depending on different applications [17].

\section{Proposed ranging and communication system}

\subsection{Principle of the system}

The original idea of ranging and communication using the SS waveform was proposed by Mizui et al. [9]. However, they just used a laser at the transmitter end and a single PD at the receiver end, which will bring the problems as discussed before: costly, bulky, requiring a fine scanning mechanism, and complicated tracking algorithm. Furthermore, it can only measure the distance instead of the location and only support point-to-point operation rather than between multiple vehicles. We will compare the modified system with the originally proposed one.

Figure 1 demonstrates the block diagram of the proposed ranging and communication system. First, vehicle no. 1 transmits a pulsed SS signature waveform $s_{1}(t)$ to vehicle no. 2. Then, vehicle no. 2 captures the signal filtered by an image lens and renews its neighbor list table (Table 1). After this, vehicle no. 2 multiplies information bits (SS modulation) to the signature waveform and
Table 1 Neighbor list table of vehicle no. 2

\begin{tabular}{lll}
\hline Neighbor & Position & Distance \\
\hline$s_{1}(t)$ & $\left(x_{1}, y_{1}\right)$ & $d_{1}$ \\
$\ldots$ & $\ldots$ & $\ldots$ \\
$s_{k}(t)$ & $\left(x_{k}, y_{k}\right)$ & $d_{k}$ \\
$\ldots$ & $\ldots$ & $\ldots$ \\
\hline
\end{tabular}

transmits back to vehicle no. 1 . Vehicle no. 1 acquires the distance measurement by sensing the time of flight (TOF) between the emitted light and received light and then extracts the information bits.

This system can work under two different modes. Even though vehicle no. 2 is not equipped with this system, vehicle no. 1 can still acquire the distance via the reflecting signal.

In this process, vehicle no. 1 can determine not only the distance but also the location of vehicle no. 2, because the imaging method can provide angle information of the incoming light. And if two or more users receive the ranging waveform, then they respond to it simultaneously, and their signal will be imaged to different areas of the PD array. The interference between different users can be mitigated significantly. The reflecting signal power falling into the corresponding $\mathrm{PD}$, which mainly senses the active responding signal, is also limited in the case that only one single user responds.

\subsection{Optical wireless communication network}

$N$ different users can be allocated $N$ different unique SS codes with the length $N$. In the mobile communication network applying code division multiple access (CDMA), the users' SS codes are assigned by the base station. In our system, the SS code is randomly generated by the user itself with the fixed length $N$. So it is possible that two

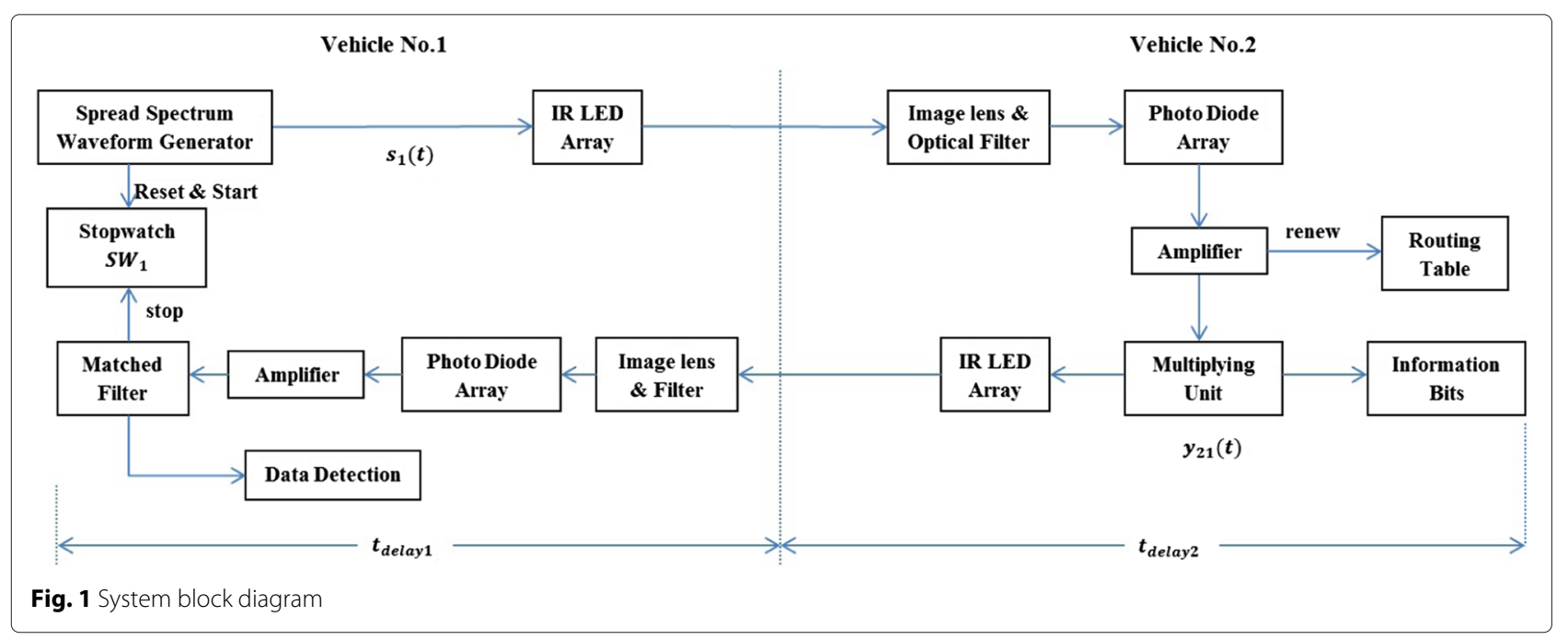


or more users with the same SS code conflict. Assume there are $n$ users in our network and the SS code length is $N$, then the probability of no SS code conflict is given by Eq. 2.

$$
P=\frac{N !}{N^{n}(N-n) !}
$$

where $N$ ! means the factorial of $N$.

Figure 2 illustrates the probability with different users $n$ and different SS code lengths $N$. We can see that the probability of no two or more users are using the same SS code decreases very quickly as the number of users in the network increases, even with a large code length $N$. Conflict of SS code will degrade ranging accuracy and communication performance. A mechanism is needed to reduce the conflict.

In order to mitigate the negative effect caused by the limited number of available SS codes, we proposed the following method to decrease the conflict probability:

1. Before sending its own SS code, the node will listen to other users and collect the SS codes being used. Then, a new SS code is generated.

2. If one user finds there are two or more users using the same code, it will inform all of these users and these users will generate new SS codes not being used.

3. For ranging application, the ranging interval of different users is the same. But the start time is random for each user and a guard time window is used. The received ranging signal beyond this window will be discarded.

4. For communication application, not only the SS code but also the location information will be used to distinguish different users.

Also, several certain SS codes should be reserved for special applications. For example, some SS codes should be assigned to broadcast emergency information. And these codes are pre-known to all users so they can demodulate the information correctly.

Another problem we need to take into consideration is the interference between the ranging process and the communication process. Although the spatial diversity and the code diversity have been applied to distinguish different users, the communication signal still can corrupt the ranging signal for the same user because these two processes use the same SS code. Thanks to the imagingbased receiving method and the 2-D PD array, only several certain PDs are corrupted. The reflecting ranging signal can still reach on most of PDs and the distance information can be acquired. Also, the computer vision technique can be applied to compensate the corrupted ranging information.

The optical communication links mainly depend on the LOS path and can be regarded as highly directional transmission. These characters make it beneficial in dealing with the broadcast problem. The broadcast information flows in a certain direction and will not flow back, which will help mitigate the broadcast problem occuring in the RF method.

\section{Experiment results}

In this section, we will report the experiment results to confirm that the proposed system can work effectively and is robust to ambient light noise, reflection, and user interference.

Figure 3 illustrates the experiment block diagram. On the left end of the diagram, an $\mathrm{M}$ sequence was generated by an arbitrary waveform generator (AWG) to drive the LED with the on-off key modulation. The length of the $M$ sequence is 31 , and the chip width is $100 \mathrm{~ns}$. A single PD was applied to capture the active responding signal with an imaging lens to collect the energy. The captured signal was recorded by an oscilloscope and then processed by MATLAB offline. On the right end, another single PD was used to receive the ranging signal. The signal was

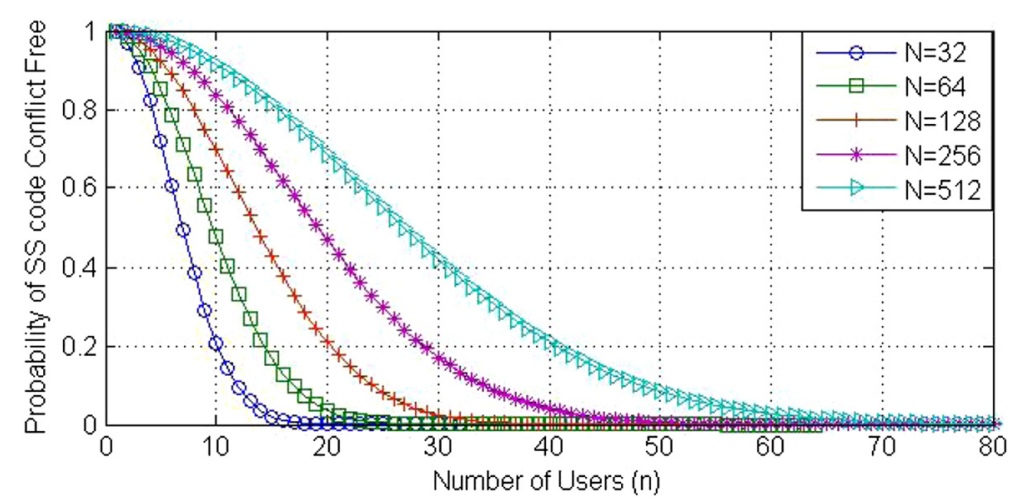

Fig. 2 SS code non-conflict probability with different code lengths $N$ 


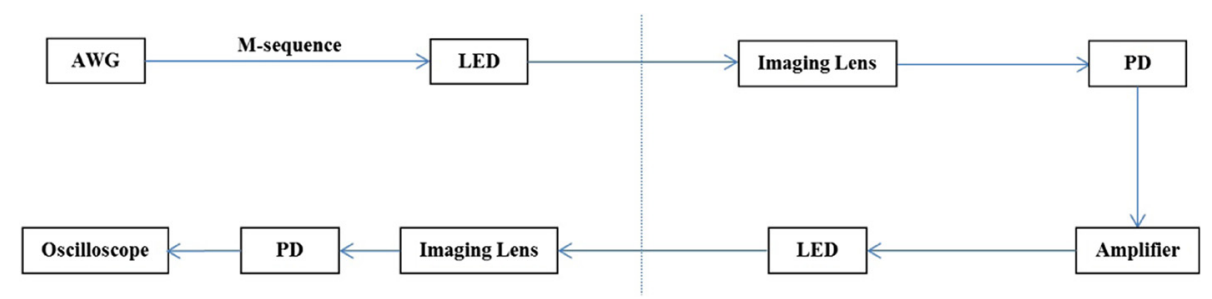

Fig. 3 Experiment block diagram

then transmitted back to the left end after being amplified. A customized PD array was not available, so we used a single PD instead. But we can still prove that the imaging method and PD array structure are beneficial later in this experiment.

The experiment was completed indoors with all office artificial lights on as the ambient light noise. The two terminals were separated at distances of $5,10,15,20,25$, and $30 \mathrm{~m}$. At each distance, ten measurements were conducted. And after $20 \mathrm{~m}$, a huge glass of about $25 \mathrm{~m}$ far behind the receiver was applied as the reflection interference. So the data acquired after $20 \mathrm{~m}$ contains the reflection noise signal. Table 2 gives the measured average time of flight at each distance which is the true time of flight plus circuit delay.

We cannot directly use these data to calculate the absolute distance because the measured time contains circuit delay. So we calculate the relative difference between two different distances instead of the absolute distance. Here we assume that the circuit delay remains the same at different distances. Table 3 shows the obtained results and deviations.

From the table, we can find there is a large deviation (more than $15 \%$ ) from 20 to $25 \mathrm{~m}$; this is because the glass brings in reflection noise after $20 \mathrm{~m}$. The reflection noise was canceled out again, so the deviation decreases to $3.2 \%$ from a distance of $25-30 \mathrm{~m}$. Except this point, we can see that the proposed system is robust to the ambient light noise. The PD was set in the focal plane of the lens. The lens before the PD focuses light into a small light spot. The PD could not detect the transmitted signal once the focused light spot was not imaged to the PD sensor area. So if you use another LED to simulate the different user interference, it will be imaged to the outside of the PD sensor area and will not affect the received signal. But if we use a PD array as the receiver, it will be imaged to another PD and demodulated correctly. This illustrates that our proposed system is also robust to the reflection interference and user interference.

\section{Conclusions}

In this paper, we developed a vehicular optical system which can acquire information and location simultaneously. The LED array which served as the transmitter can make this system less expensive and compact. The PD array based on the imaging receiving method can provide high lateral resolution, while keeping a wide FOV without a scanning mechanism. From the experiment results, we can see this system can greatly suppress the user interference if the incoming light from other source is being imaged to the area out of the PD sensor. However, the reflection interference from large background objects, when they are in the same path as our target, may more or less affect the ranging precision, which we could see from row 5 in Table 3. Imaging processing or other techniques could be applied to mitigate such reflection noise in the future. Also, for a customized PD array, an efficient algorithm should be proposed to detect the effective PDs, where the signal is being sensed. And last, the network protocol for the vehicular optical network system is what we will discuss next in the future.
Table 2 Measured average TOF for different distances

\begin{tabular}{ll}
\hline Distance $(\mathrm{m})$ & Average TOF (ns) \\
\hline 5 & 99.56 \\
10 & 117.33 \\
15 & 134.19 \\
20 & 151.62 \\
25 & 165.65 \\
30 & 182.85 \\
\hline
\end{tabular}

Table 3 Measured distance difference results

\begin{tabular}{lll}
\hline Distance difference $(\mathrm{m})$ & Measured distance $(\mathrm{m})$ & Deviation $(\%)$ \\
\hline $5-10$ & 5.331 & 6.62 \\
$10-15$ & 5.058 & 1.16 \\
$15-20$ & 5.229 & 4.58 \\
$20-25$ & 4.209 & 15.82 \\
$25-30$ & 5.160 & 3.2 \\
\hline
\end{tabular}




\section{Competing interests}

The authors declare that they have no competing interests.

Received: 31 October 2014 Accepted: 12 June 2015

Published online: 14 July 2015

\section{References}

1. U.S. Department of Transportation, Vehicle Safety Communications Project Task 3 Final Report. http://www.ntis.gov. Accessed 4 April 2014

2. Dedicated short range communications (DSRC). http://www.its.dot.gov/ DSRC/. Accessed 4 April 2014

3. M Fukuzawa, H Hama, N Nakamori, M Yamada, in Computer and Information Technology, 2008. ICCIT 2008. 11th International Conference On. High-speed distance measurement between moving vehicles with NIR-LED markers (Khulna, 24-27 Dec. 2008), pp. 516-520

4. N Stone, J Billingsley, Development of low cost range sensing instrumentation for collision avoidance applications. Mechatron. Mach. Vision. Robot. Mechatron. 3, 127-133 (2000)

5. O Elkhalili, OM Schrey, W Ulfig, W Brockherde, BJ Hosticka, P Mengel, L Listl, in Solid-State Circuits Conference, 2006. ESSCIRC 2006. Proceedings of the 32nd European. A 64x8 pixel 3-D CMOS time of flight image sensor for car safety applications (Montreaux, Switzerland, Sept. 2006), pp. 568-571

6. K Cui, G Chen, Z Xu, RD Roberts, in GLOBECOM Workshops (GC Wkshps), 2011 IEEE. Experimental characterization of traffic light to vehicle VLC link performance, (2011), pp. 808-812

7. SJ Lee, JK Kwon, SY Jung, YH Kwon, in Intelligent Transportation Systems (ITSC), 2012 15th International IEEE Conference On. Simulation modeling of visible light communication channel for automotive applications (Anchorage, AK, 16-19 Sept. 2012), pp. 463-468

8. CB Liu, B Sadeghi, EW Knightly, in Proceedings of the Eighth ACM International Workshop on Vehicular Inter-networking. VANET'11. Enabling vehicular visible light communication (V2LC) networks (Las Vegas, NV, USA, 19-23 September 2011), pp. 41-50. doi:10.1145/2030698.2030705

9. K Mizui, M Uchida, M Nakagawa, in Vehicular Technology Conference, 1993., 43rd IEEE. Vehicle-to-vehicle communication and ranging system using spread spectrum technique (proposal of boomerang transmission system) (Secaucus, NJ, 18-20 May 1993), pp. 335-338

10. S Masuda, K Mizui, in Vehicular Technology Conference, 1998. VTC 98. 48th IEEE. Vehicle-to-vehicle communication and location system using spread spectrum technique, vol. 3 (Ottawa, Ontario, 18-21 May 1998), pp. $1775-17793$

11. K Mizui, M Nakagawa, in Electrical and Computer Engineering, 1998. IEEE Canadian Conference On. Vehicle-to-vehicle multi-target communication and ranging system using spread spectrum technique, vol. 1 (Waterloo, Ontario, 24-28 May 1998), pp. 425-4281

12. K Mizui, in Intelligent Transportation Systems, 2002. Proceedings. The IEEE 5 th International Conference On. Inter-vehicle communication and ranging system using FH/SS technique (Singapore, 6 Sept. 2002), pp. 313-317

13. A Ueda, K Mizui, in Electrical and Computer Engineering, 2002. IEEE CCECE 2002. Canadian Conference On. Vehicle-to-vehicle communication and ranging system using code-hopping spread spectrum technique with code collision avoidance algorithm, vol. 3 (Winnipeg, Manitoba, Canada, 12-15 May 2002), pp. 1250-12543

14. RD Richmond, SC Cain, Direct-Detection LADAR Systems. (SPIE Press, tutorial text, Bellingham, 2010)

15. R Miyagawa, T Kanade, CCD-based range-finding sensor. IEEE Trans. Electron Devices. 44(10), 1648-1652 (1997)

16. A Ashok, M Gruteser, N Mandayam, J Silva, M Varga, K Dana, in Proceedings of the Sixteenth Annual International Conference on Mobile Computing and Networking. Challenge: mobile optical networks through visual MIMO (Chicago, Illinois, USA, 20-24 September 2010), pp. 105-112

17. JS Kwak, JH Lee, Infrared transmission for intervehicle ranging and vehicle-to-roadside communication systems using spread-spectrum technique. IEEE Trans. Intell. Transp. Syst. 5(1), 12-19 (2004)

\section{Submit your manuscript to a SpringerOpen ${ }^{0}$ journal and benefit from:}

- Convenient online submission

- Rigorous peer review

- Immediate publication on acceptance

- Open access: articles freely available online

- High visibility within the field

- Retaining the copyright to your article

Submit your next manuscript at $\boldsymbol{\wedge}$ springeropen.com 